\title{
Cooperative damping mechanism of the resonance in the nuclear photoabsorption
}

\author{
M. Hirata, K. Ochi \\ Department of physics, Hiroshima University, Higashi-Hiroshima 739, Japan \\ and \\ T. Takaki \\ Onomichi Junior College, Onomichi 722, Japan
}

\begin{abstract}
We propose a resonance damping mechanism to explain the disappearance of the peaks around the position of the resonances higher than the $\Delta$ resonance in the nuclear photoabsorption. This phenomenon is understood by taking into account the cooperative effect of the collision broadening of $\Delta$ and $N^{*}$, the pion distortion and the interference in the two-pion photoproduction processes in the nuclear medium.
\end{abstract}

PACS number(s): 25.20.Lj, 14.20.Gk, 24.30.Gd 
The total nuclear photoabsorption cross section has been measured on ${ }^{238} \mathrm{U}$ [1], Be, $\mathrm{C}$ [2], ${ }^{7} \mathrm{Li}, \mathrm{C}, \mathrm{Al}, \mathrm{Cu}, \mathrm{Sn}$ and $\mathrm{Pb}$ [3]. The data show that the excitation peaks around the position of the $D_{13}(1520)$ and $F_{15}(1680)$ resonances disappear and above $600 \mathrm{MeV}$ there is a reduction of the absolute value of the cross section per nucleon compared with the data for hydrogen [4, 5] and deuteron [6]. These experimental findings have been confirmed by the contemporary data on the photofission cross section of ${ }^{238} \mathrm{U}[\mathrm{]}]$ and ${ }^{235} \mathrm{U}$ [8] obtained at Mainz up to $800 \mathrm{MeV}$.

Some theoretical calculations based on simplified models have been attempted to explain the strong damping of the peaks around the location of the $D_{13}$ and $F_{15}$ resonances [9, 10]. In both of attempts, the collision broadening of the resonances has been assumed to be dominant damping mechanism and very large resonance widths compared with the free ones were found to be needed to reproduce the experimental data. However, the collision broadening has been estimated using the interaction of the resonance with the nucleon in Ref. [11] where it has been claimed that such significantly increasing resonance widths were hardly justified. These theoretical analyses must miss some important effects other than the collision broadening.

The purpose of this letter is to discuss a mechanism for the damping phenomena from a qualitative point of view. Here we focus only on the $D_{13}\left(N^{*}\right)$ resonance region. In order to get an idea what is going on in this energy region, it is necessary to know the details of the elementary process in the nuclear photoabsorption. The dominant reactions on a nucleon over the photon energy range from 500 to $850 \mathrm{MeV}$ are the one-pion and two-pion photoproduction [12]. The peak of the $D_{13}$ resonance region shows up clearly by the combined effect of these reactions as can be seen in Fig. 1 where the cross section of the one-pion photoproduction has a small peak at around $720 \mathrm{MeV}$ and the cross section of the two-pion photoproduction starts to grow from around $400 \mathrm{MeV}$ photon energy and increases up to around $800 \mathrm{MeV}$. The $D_{13}$ resonance plays an important role in the total cross sections of these reactions. In the one-pion photoproduction, the effect of the $P_{33}(1232)$ resonance diminishes with increasing energy in this energy region and instead the $D_{13}$ resonance contributes dominantly to produce the small peak of the cross section at around $720 \mathrm{MeV}$. The latter is due to that the $D_{13}$ resonance has a large photocoupling, the $\pi N$ branching fraction of 50-60\% and a width comparable to the $P_{33}(1232)$ resonance. Its photocoupling is at least twice larger than those of the other relevant resonances such as $P_{11}(1440), S_{11}(1535)$ and $P_{33}(1600)$ [13]. In our present approach which will be described later, therefore, the partial wave amplitudes other than $P_{33}$ and $D_{13}$ are regarded as background contributions. In the two-pion photoproduction, the production processes through the excitation of a resonance by $\gamma N$ coupling [e.g., see Figs. 2(a) and 2(b)] have themselves small contributions and the dominant contributions are due to the production processes through the $\Delta$ Kroll-Ruderman, the $\Delta$ pion-pole and the $\rho$ Kroll-Ruderman terms [Figs. 2(c), 2(d) and 2(e)]. The interference between the $D_{13}$ resonance term and the Kroll-Ruderman terms, however, is essential to produce the bump of the cross section [14 16. Thus the contribution of the $D_{13}$ resonance term is important compared with the other resonances. We take into account only the $D_{13}$ resonance in the resonances excited by $\gamma N$ coupling.

For the one-pion photoproduction, the analyses due to the partial wave expansion had been carried out by several authors [17]. The cross sections for both proton and neutron can be obtained by using partial wave amplitudes given by them. In our calculation we use 
the SM95 amplitudes of Arndt et al. [17]. In the case of proton only a small peak appears near the resonance energy of $D_{13}$, but in the case of neutron the energy dependence of cross section near the same energy shows a shoulder rather than a peak. This behavior of the $D_{13}$ resonance is quite different from that of the $\Delta$ resonance. Therefore, we can easily make the $D_{13}$ resonance peak for the one-pion photoproduction vanish by introducing much smaller width due to the collision broadening than those given in Refs. [9,10].

The two-pion photoproduction has been studied by Tejedor and Oset [14], and Murphy and Laget [15]. The models have been successful to predict the $\gamma p \rightarrow \pi^{+} \pi^{-} p$ cross section. However, predicted cross sections of the $\gamma p \rightarrow \pi^{+} \pi^{0} n$ and $\gamma n \rightarrow \pi^{-} \pi^{0} p$ reactions are too small compared with the experimental data [12,18]. The total photoabsorption cross section on a nucleon cannot be reproduced by using their models. For the present purpose we need the model which gives a good description of the two-pion photoproduction. Recently we have proposed a simple model to reproduce the total cross section of two-pion photoproduction [16]. In this model, $N^{*}, \Delta$ and $\rho$ meson are treated in a separable potential model which is able to describe the resonance over a wide range of energy [19]. The total photoabsorption cross sections on both the proton and the neutron target can be reproduced over the photon energy range between $400 \mathrm{MeV}$ and $850 \mathrm{MeV}$ by using this model [16], e.g., see Fig. [1. Hereafter we discuss the two-pion photoproduction based on our model.

The two-pion photoproduction takes place through the $\pi \Delta$ or the $\rho N$ channel. The $N^{*}$ resonance decays into a $\pi \pi N$ state through these channels which diagrams are shown in Figs. 2(a) and 2(b). The corresponding $T$ matrices are denoted by $T_{N^{*}(\pi \Delta)}^{s(d)}$ and $T_{N^{*} \rho N}$ respectively, where $s(d)$ means a $s$-wave or $d$-wave $\pi \Delta$ state. In the $\gamma p \rightarrow \pi^{+} \pi^{-} p$ reaction, the dominant processes are the $\Delta$ Kroll-Ruderman term $\left(T_{\Delta \mathrm{KR}}\right)$ in Fig. 2 (c) and $\Delta$ pionpole term $\left(T_{\Delta \mathrm{PP}}\right)$ in Fig. 2(d). The $N^{*}$ contribution alone is small but the interference between $T_{N^{*}(\pi \Delta)}$ and $T_{\Delta \mathrm{KR}}$ is important. Because of this, the $N^{*}$ excitation is regarded as an important ingredient in the two-pion photoproduction. In the $\gamma p \rightarrow \pi^{+} \pi^{0} n$ reaction, the $\rho$ Kroll-Ruderman term $\left(T_{\rho \mathrm{KR}}\right)$ and the $N^{*}$ terms dominate. In this case, the interference among $T_{\Delta \mathrm{KR}}, T_{\rho \mathrm{KR}}$ and $T_{N^{*} \rho N}$ is important and gives rise to the bump in the cross section. So, we expect that the delicate balance of the interference is broken in the nuclear medium by the collision broadening of the $\Delta$ and the $N^{*}$ and the pion distortion in the $\pi \Delta$ channel and therefore the bump is strongly damped due to the cooperative effect of the broadening and the interference.

Now let us investigate the strong damping in the nuclear photoabsorption. For simplicity we use the Fermi gas model for a nucleus. The absorption processes are assumed to consist of three components: the quasi-free processes such as the one-pion photoproduction and two-pion photoproduction, and genuine many-body absorption processes arising from the interaction between the resonance (or pion) and the nucleon in a nucleus [20].

In the laboratory frame the cross section of one-pion photoproduction off a proton in the nuclear matter is given by

$$
\begin{aligned}
\sigma_{p}^{\pi}= & \frac{1}{v} \frac{3 Z}{8 \pi\left(k_{f}^{p}\right)^{3}} \int_{0}^{k_{f}^{p}} d \vec{p}_{1} \int \frac{d \vec{q}}{(2 \pi)^{3}} \frac{d \vec{p}}{(2 \pi)^{3}}(2 \pi)^{4} \delta^{4}\left(k+p_{1}-q-p\right) \\
& \times \frac{1}{2} \sum_{\lambda, \nu, \nu^{\prime}} \sum_{t_{\pi}, t_{N}}\left|<\vec{q} t_{\pi} \vec{p} \nu^{\prime} t_{N}\right| T_{B}+T_{N^{*}}+T_{\Delta}\left|\vec{k} \lambda \vec{p}_{1} \nu>\right|^{2} \theta\left(|\vec{p}|-k_{f}^{t_{N}}\right) \frac{M^{2}}{2 k 2 \omega_{\vec{q}} E_{\vec{p}} E_{\vec{p}}},
\end{aligned}
$$

where $T_{N^{*}}$ and $T_{\Delta}$ represent the $N^{*}$ and $\Delta$ resonance terms, respectively and $T_{B}$ is the 
background term. $\vec{k}, \overrightarrow{p_{1}}, \vec{q}$ and $\vec{p}$ are the momenta of the incident photon, target proton, outgoing pion and outgoing nucleon, respectively. $E_{\vec{p}}, \omega_{\vec{q}}$, and $E_{\vec{p}}$ are the energies of the target proton, outgoing pion, and outgoing nucleon, respectively. $Z$ and $v$ denote the proton number and the relative velocity between the photon and nucleus, respectively. $k_{f}^{t_{N}}$ is the Fermi momentum depending on the isospin quantum number $t_{N}$. The notation for all other spin-isospin quantum numbers are self-explanatory. The $N^{*}$ resonance term is expressed as

$$
\begin{aligned}
T_{N^{*}} & =F_{\pi N^{*}} G_{N^{*}}(s) \tilde{F}_{\gamma P N^{*}}^{\dagger}, \\
G_{N^{*}}(s) & =\left[\sqrt{s}-\left(M_{N^{*}}(s)+\delta M_{N^{*}}\right)+i\left(\Gamma_{N^{*}}(s)+\Gamma_{N^{*} s p}\right) / 2\right]^{-1},
\end{aligned}
$$

where $\sqrt{s}$ is the total center of mass energy. $M_{N^{*}}$ and $\Gamma_{N^{*}}$ in the $N^{*}$ propagator $G_{N^{*}}$ are the mass and the free width of $N^{*}$, respectively, which are given so as to describe the energy dependence of the $\pi N D_{13}$-wave scattering amplitude and the branching ratios at the resonance energy. $\delta M_{N^{*}}$ and $\Gamma_{N^{*} s p}$ are the mass shift and spreading width due to the collisions between $N^{*}$ and other nucleons, respectively. $F_{\gamma P N^{*}}^{\dagger}$ and $F_{\pi N N^{*}}$ are the $\gamma P N^{*}$ and $\pi N N^{*}$ vertex functions, respectively, of which detail forms are given in Ref. [16]. The $\Delta$ resonance term $T_{\Delta}$ is written in a similar form with $T_{N^{*}}$ but is important only at the low energy range less than $500 \mathrm{MeV}$. The Pauli blocking effect for the $\Delta$ decay into $\pi N$ becomes non-negligible at low energies, since the probability of the nucleon being emitted with a small momentum increases compared with the energy region of the $N^{*}$ resonance [20,21]. Thus, we include the Fock term in the $\Delta$ propagator to modify the free $\Delta$ self-energy in addition to the collision width. The integration over final particle momenta in Eq. (1) is performed by using variables defined in the $\gamma N$ center of mass system. We will also calculate the cross section of two-pion photoproduction in the same way.

The cross section of the two-pion photoproduction off a proton is given by

$$
\begin{aligned}
\sigma_{p}^{2 \pi}= & \frac{1}{v} \frac{3 Z}{8 \pi\left(k_{f}^{p}\right)^{3}} \int_{0}^{k_{f}^{p}} d \vec{p}_{1} \int \frac{d \vec{q}_{1}}{(2 \pi)^{3}} \frac{d \overrightarrow{q_{2}}}{(2 \pi)^{3}} \frac{d \vec{p}}{(2 \pi)^{3}}(2 \pi)^{4} \delta^{4}\left(k+p_{1}-q_{1}-q_{2}-p\right) \\
& \times \frac{1}{2} \sum_{\lambda, \nu, \nu^{\prime}} \sum_{t_{\pi_{1}} t_{\pi_{2}} t_{N}}\left|<\vec{q}_{1} t_{\pi_{1}} \vec{q}_{2} t_{\pi_{2}} \vec{p} t_{N} \nu^{\prime}\right| T\left|\vec{k} \lambda \vec{p}_{1} \nu>\right|^{2} \theta\left(|\vec{p}|-k_{f}^{t_{N}}\right) \frac{M^{2}}{2 k 2 \omega_{\vec{q}_{1}} 2 \omega_{\vec{q}_{2}} E_{\vec{p}_{1}} E_{\vec{p}}},
\end{aligned}
$$

where $\vec{q}_{1}$ and $\vec{q}_{2}$ are the momenta of the outgoing pions. The medium-modified $T$ matrix is expressed as

$$
T=T_{\Delta K R}+T_{\Delta P P}+T_{N^{*} \pi \Delta}^{s}+T_{N^{*} \pi \Delta}^{d}+T_{\rho \mathrm{KR}}+T_{N^{*} \rho N} .
$$

The $\Delta$ Kroll-Ruderman term is written as

$$
\begin{aligned}
T_{\Delta K R} & =F_{\pi N \Delta} G_{\pi \Delta}\left(s, \vec{p}_{\Delta}\right) F_{\Delta K R}^{\dagger} \\
G_{\pi \Delta}\left(s, \vec{p}_{\Delta}\right) & =\left[\sqrt{s}-\omega_{\pi}\left(\vec{p}_{\Delta}\right)-\left(M_{\Delta}\left(s, \vec{p}_{\Delta}\right)+\delta M_{\Delta}\right)+i\left(\Gamma_{\Delta}\left(s, \vec{p}_{\Delta}\right)+\Gamma_{\Delta s p}\right) / 2-V_{\pi}\left(\vec{q}_{\pi}\right)\right]^{-1}
\end{aligned}
$$

Here $F_{\pi N \Delta}$, is the $\pi N \Delta$ vertex function, and $F_{\Delta \mathrm{KR}}^{\dagger}$ is the $\Delta$ Kroll-Ruderman vertex function. The detail forms of vertex functions are given in Ref. [16]. $V_{\pi}\left(\vec{q}_{\pi}\right)$ is the pion self-energy due to the distortion. $\vec{p}_{\Delta}$ is the $\gamma N$ center of mass momentum of $\Delta$ and $\vec{q}_{\pi}$ is the outgoing pion momentum. $M_{\Delta}$ and $\Gamma_{\Delta}$ in the propagator $G_{\pi \Delta}$ are the mass and the free width of $\Delta$, respectively and $\delta M_{\Delta}$ and $\Gamma_{\Delta s p}$ are the mass shift and collision width, respectively. The 
other terms in the r.h.s. of Eq. (5) are expressed in a similar way. The detail form of free $T$ matrices are given in Ref. [16].

In addition to the one- and two-pion photoproduction processes, there are three genuine many-body processes which are shown in Figs. 2(f), 2(g) and 2(h). The cross section for Fig. 2(f) is given by

$$
\sigma_{N^{*}(A-1)}^{p}=\frac{1}{v} \frac{3 Z}{8 \pi\left(k_{f}^{p}\right)^{3}} \int_{0}^{k_{f}^{p}} d \vec{p}_{1} \Gamma_{N^{*} s p} \frac{1}{2} \sum_{\lambda \nu \nu_{N^{*}}}\left|G_{N^{*}}(s)<\vec{p}_{N^{*}} \nu_{N^{*}}\right| \tilde{F}_{\gamma P N^{*}}^{\dagger}\left|\vec{k} \lambda \vec{p}_{1} \nu>\right|^{2} \frac{1}{2 k} \frac{M}{E_{\vec{p}_{1}}} .
$$

The cross section for Fig. 2(g) has a similar form with Eq. (15). The cross section for Fig. $2(\mathrm{~h})$ is given by

$$
\begin{aligned}
\sigma_{\pi \Delta(A-1)}^{p}= & \frac{1}{v} \frac{3 Z}{8 \pi\left(k_{f}^{p}\right)^{3}} \int_{0}^{k_{f}^{p}} d \vec{p}_{1} \int \frac{d \vec{q}}{(2 \pi)^{3}} \frac{d \vec{p}}{(2 \pi)^{3}}\left(\Gamma_{\Delta s p}+2 \operatorname{Im} V_{\pi}(\vec{q})\right)(2 \pi)^{3} \delta\left(\vec{k}+\vec{p}_{1}-\vec{q}-\vec{p}\right) \\
& \times \frac{1}{2} \sum_{\lambda \nu \nu_{\Delta}}\left|G_{\pi \Delta}\left(s, p_{\Delta}\right)<\vec{q} t_{\pi} \vec{p} \nu_{\Delta} t_{\Delta}\right| F_{\gamma P \pi \Delta}^{\dagger}\left|\vec{k} \lambda \vec{p}_{1} \nu>\right|^{2} \frac{1}{2 k 2 \omega_{\vec{q}}} \frac{M}{E_{\vec{p}_{1}}}
\end{aligned}
$$

where $F_{\gamma P \pi \Delta}^{\dagger}$ describes the $\gamma P \rightarrow \pi \Delta$ transition corresponding to Figs. 2(a)-2 2 (d). The cross sections of photoabsorption on a neutron in the nuclear matter are also given in a similar form with those of a proton.

In the numerical calculations, we make an approximation that the target nucleon's momentum $\vec{p}_{1}$ in the $T$-matrix elements is taken to be zero. Although the effect of Fermi motion makes the bump of the cross section small and broad, the missing strength of the cross section cannot be explained at all with its effect alone 10,11]. Therefore it is clear that one has to seek the other possibilities to understand the strong suppression. We think the above approximation is sufficient to investigate the qualitative aspect of the damping mechanism. In order to evaluate the cross sections, we need the values of the mass shifts and collision widths of $\Delta$ and $N^{*}$, and the pion self-energy $V_{\pi}$. The mass shift and collision width of $\Delta$ have already known in the studies of pion-nucleus scattering using $\Delta$-hole model [21,22] where they can be identified to the spreading potential. The spreading potential found in these studies is almost energy-independent. We take $\delta M_{\Delta}=10 \mathrm{MeV}$ and $\Gamma_{\Delta s p}=80 \mathrm{MeV}$. As the pion self-energy, we adopt the pion optical potential used by Arima et al. [23]. $V_{\pi}$ has a strong energy-dependence because the pion-nucleon interaction in the relevant energy region is dominated by the $\Delta$ resonance. As for the mass shift and collision width of $N^{*}$, i.e., $\delta M_{N^{*}}$ and $\Gamma_{N^{*} s p}$, there are no established values at present. For simplicity, we assume that $\delta M_{N^{*}}$ and $\Gamma_{N^{*} s p}$ are energy-independent like $\delta M_{\Delta}$ and $\Gamma_{\Delta s p}$. Furthermore, we choose these values so that the total nuclear photoabsorption cross sections from $600 \mathrm{MeV}$ to $800 \mathrm{MeV}$ are reproduced. We found $\delta M_{N^{*}}=-20 \mathrm{MeV}$ and $\Gamma_{N^{*} s p}=80 \mathrm{MeV}$, where the collision width of $N^{*}$ is the same as that of $\Delta$. The calculated total photoabsorption cross sections per nucleon (thick solid line) are shown in Fig. 3. In this calculation we use the Fermi momentum $k_{f}$ obtained using the central nuclear density and found that the Pauli blocking effect is very small $(\leq 5 \%)$ above $500 \mathrm{MeV}$. We also show each contribution of the absorption processes: the one-pion photoproduction (dashed line), the two-pion photoproduction (dotted line), the many-body absorption through the $\Delta$-nucleus state and $N^{*}$-nucleus state (dash-dotted line) corresponding to Figs. 2(f) and 2(g) and the many-body absorption through the $\pi \Delta$-nucleus 
state (long dashed line) corresponding to Fig. 2(h). For comparison, our theoretical result (thin solid line) for the total photoabsorption cross section on a proton is shown in Fig.3.

In the one-pion photoproduction, the bump near the mass of the $N^{*}$ disappears by the effect of the spreading potential for the $N^{*}$. The cross sections of the two-pion photoproduction are quite suppressed by the cooperative effect between the following medium corrections: the spreading potentials for the $\Delta$ and the $N^{*}$, the pion distortion and the change of the interference among the related reaction processes. The cross sections of the other many-body processes are almost flat in the energy range above $600 \mathrm{MeV}$ and small. As a consequence of these effects, the excitation peak around the position of the $D_{13}$ resonance in the total nuclear photoabsorption cross sections disappear differently from the hydrogen. Extremely large collision width is not necessary for the $N^{*}$ resonance. Since the Fermi motion broadens the resonance peak and suppresses its value, the inclusion of its effect in our calculation would probably lead to a smaller collision width than the value obtained in our approach.

In order to confirm our conclusion and get more precise information regarding the mass shift and the collision broadening for the $N^{*}$, it is necessary to take into account the Fermi motion of the nucleon in our calculation. This analysis is in progress. Its results and the formalism used in this letter will be reported in detail elsewhere. 


\section{REFERENCES}

[1] N. Bianchi et al., Phys. Lett. B 229, 219 (1993).

[2] M. Anghinolfi et al., Phys. Rev. C 47, R992 (1993).

[3] N. Bianchi et al., Phys. Lett. B 309, 5 (1993); Phys. Lett. B 325, 333 (1994); Phys. Rev. C 54, 1688 (1996).

[4] T.A. Armstrong et al., Phys. Rev. D 5, 1640 (1972).

[5] M. MacCormick et al., Phys. Rev. C 53, 41 (1996).

[6] T.A. Armstrong et al., Nucl. Phys. B41, 445 (1972).

[7] Th. Frommhold et al., Phys. Lett. B295, 28 (1992).

[8] Th. Frommhold et al., Z. Phys. A350, 249 (1994).

[9] W.M. Alberico, G.Gervino,and A.Lavagno, Phys. Lett. B321, 177 (1994).

[10] L.A. Kondratyuk et al., Nucl. Phys. A579, 453 (1994).

[11] M. Effenberger, A. Hombach, S. Teis, and U. Mosel, Nucl. Phys. A613, 353 (1997).

[12] A. Braghieri et al., Phys. Lett. B363, 46 (1995).

[13] Particle Data Group, Phys. Rev. D54, 1 (1996).

[14] J. A. G. Tejedor and E. Oset, Nucl. Phys. A571, 667 (1994); Nucl. Phys. A600, 413 (1997).

[15] L.Y. Murphy and J.M. Laget, Report No. CEA/DAPNIA-SPhN 95-42.

[16] K. Ochi, M. Hirata, and T. Takaki, Phys. Rev. C 56, 1472 (1997).

[17] R.A. Arndt, I.I. Strakovsky, and R.L. Workman, Phys. Rev. C56, 577 (1997) and references therein.

[18] A. Zabrodin et al., Phys. Rev. C55, 1 (1997).

[19] M. Betz and T.-S.H. Lee, Phys. Rev. C23, 375 (1981).

[20] J.H. Koch, E.J. Moniz, and N. Ohtsuka, Ann. Phys. (N.Y.) 154, 99 (1984).

[21] Y. Horikawa, F. Lenz, and M. Thies, Nucl. Phys. A345, 386 (1980).

[22] E. Oset and W. Weise, Nucl. Phys. A 319, 477 (1979); A 329, 365 (1979).

[23] M. Arima, K. Masutani, and R. Seki, Phys. Rev. C 51, 285 (1995). 


\section{FIGURES}

FIG. 1. The total photoabsorption cross section on a proton. The dashed line is obtained by using SM95 amplitudes of Arndt et al. [17]. Solid and dash-dotted lines are theoretical predictions by our model [16]. Solid circles represent the data of total photoabsorption cross section on a proton [5. Open squares represent the data of two-pion photoproduction cross section [2].

FIG. 2. Diagrams for the two-pion photoproduction on a nucleon and genuine many-body absorption processes on a nucleus. (a) The $N^{*} \rightarrow \pi \Delta$ contribution. (b) The $N^{*} \rightarrow \rho N$ contribution. (c) The $\Delta$ Kroll-Ruderman term. (d) The $\Delta$ pion-pole term. (e) The $\rho$ Kroll-Ruderman term. (f) The many-body absorption process through the $N^{*}$. (g) The many-body absorption process through the $\Delta$. (h) The many-body absorption process through the $\pi \Delta$. A is the mass number of the target nucleus.

FIG. 3. The total nuclear photoabsorption cross section on nuclei. The thick solid line is the full calculation. The dashed and dotted lines are the contributions of the one-pion and two-pion production, respectively. The dash-dotted line is the contribution for the processes of Figs. 2(f) and 2(g). The long dashed line is the contribution for the process of Fig. 2(h). The thin solid line is the theoretical result for the total cross section on a proton calculated by our model. Experimental data are taken from Ref. [3] 


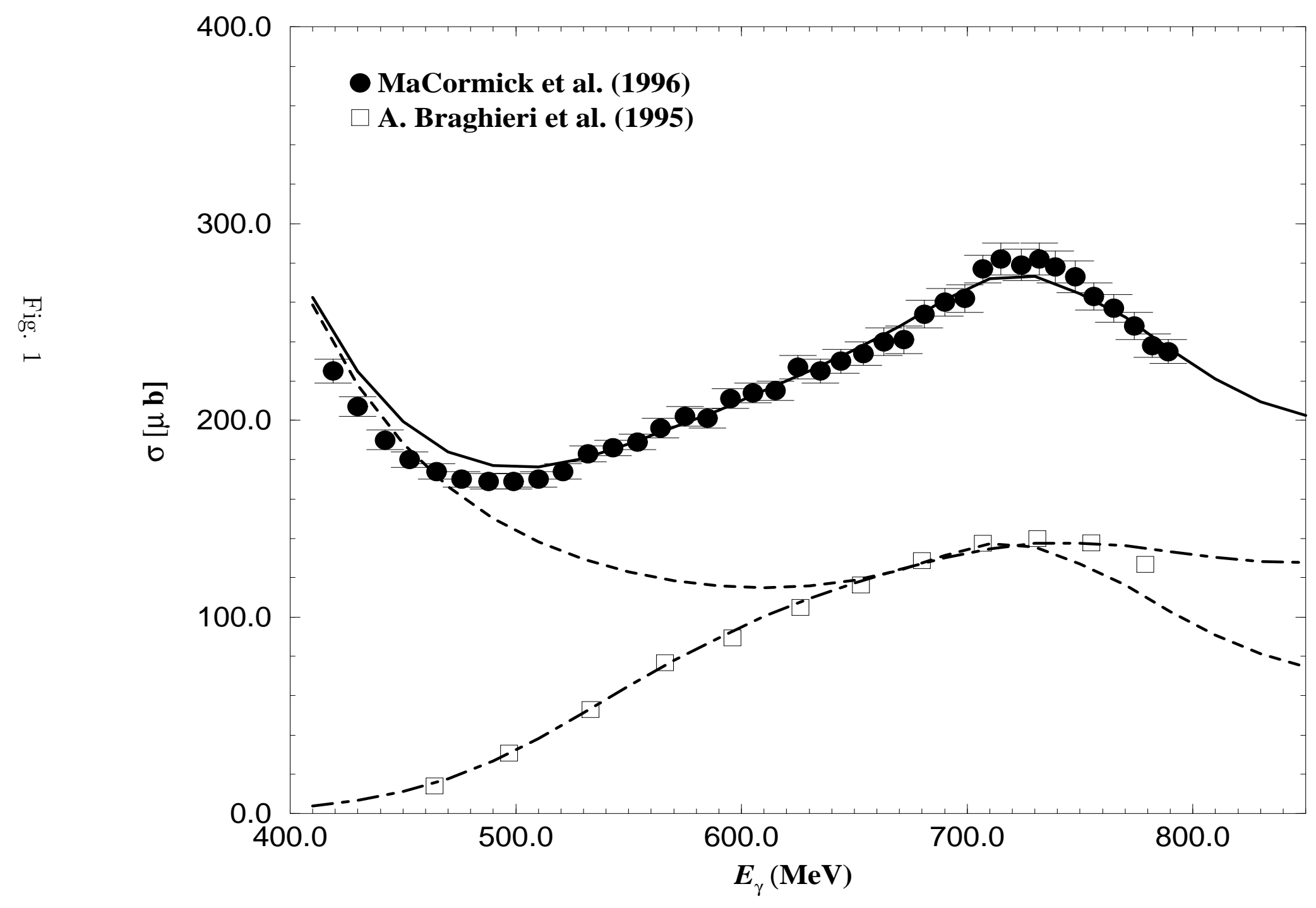




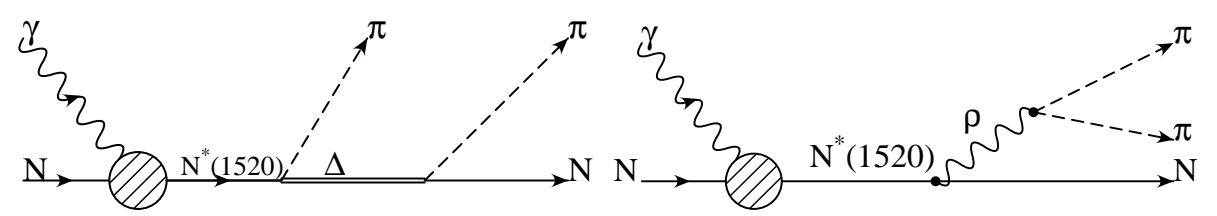

(a)

(b)

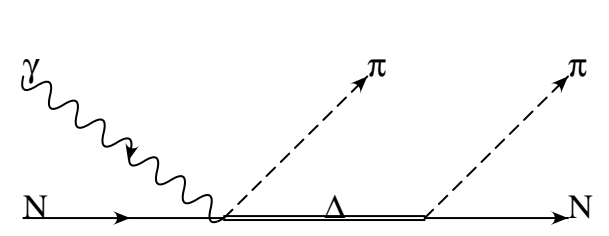

(c)

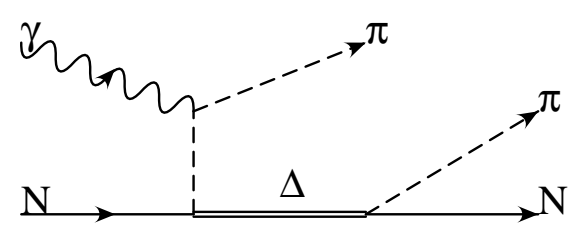

(d)

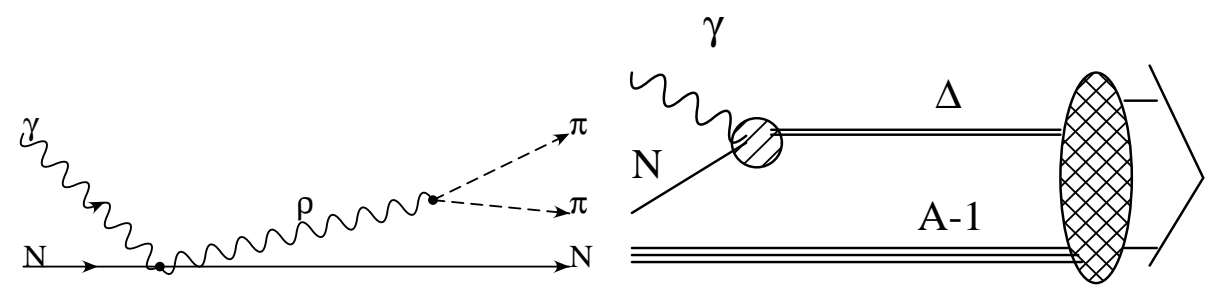

(e)

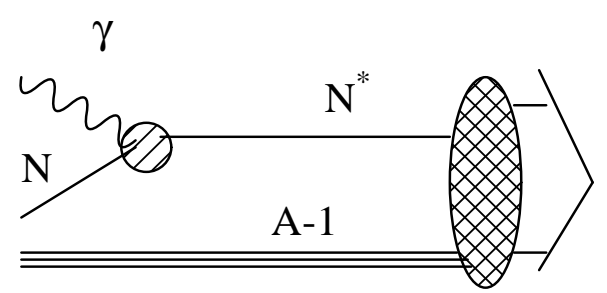

(g)

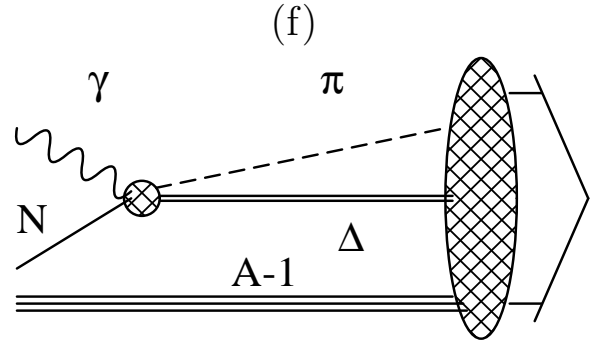

(h)

Fig. 2 


\section{$\gamma A$}



